NASA Technical Memorandum 102487

\title{
Steady-State and Transient Zener Parameters in Viscoplasticity: Drag Strength Versus Yield Strength
}

\author{
A.D. Freed \\ Lewis Research Center \\ Cleveland, Ohio \\ and \\ K.P. Walker \\ Engineering Sciencè Software \\ Smithfield, Rhode Island
}

Prepared for the

11th U.S. National Congress of Applied Mechanics

Tucson, Arizona, May 21-25, 1990 

$\because=$

- 


\title{
STEADY-STATE AND TRANSIENT ZENER PARAMETERS IN VISCOPLASTICITY:
}

\section{DRAG STRENGTH VERSUS YIELD STRENGTH}

\author{
A.D. Freed \\ National Aeronautics and Space Administration \\ Lewis Research Center \\ Cleveland, Ohio 44135
}

and

\author{
K.P. Walker \\ Engineering Science Sof tware \\ Smithfield, Rhode Island 02917
}

\begin{abstract}
A hypothesis is put forth which enables the viscoplastician to formulate a theory of viscoplasticity that reduces, in closed form, to the classical theory of creep. This hypothesis is applied to a variety of drag and yield strength models. Because of two theoretical restrictions that are a consequence of this hypothesis, three different yield strength models and one drag strength model are shown to be theoretically admissible. One of these yield strength models is selected as being the most appropriate representation for isotropic hardening.
\end{abstract}

\section{INTRODUCTION}

Over the past two decades, development of internal state variable theories of viscoplasticity has been an active topic of research ( $c f$. Freed and Chaboche (1989) for a bibliography). Much of this activity has been centered around what the evolution equations for internal state should look like. This paper attempts to address a more fundamental question. There are two different isotropic variables that can be found in the various viscoplastic models published in the literature; they are, the drag strength and the yield strength. Which one of these variables provides the most appropriate representation of isotropic hardening? An objective of this paper is to answer that question. Another objective is to formulate a viscoplastic theory that reduces, in closed form, to the classical theory of creep at steady state. In our efforts to meet the second objective, the first objective is also met.

\section{ELASTIC-VISCOPLASTIC CONTINUA}

Small material displacements and rotations are assumed to make up the deformation of an elastic-viscoplastic continuum. In a Cartesian reference configuration, the infinitesimal strain $\varepsilon_{i j}$ is taken to be composed of elastic $\varepsilon_{i j}^{e}$ (thermodynamically reversible - includes thernal strain) and inelastic or plastic $\varepsilon_{i j}^{p}$ (thermodynamically irreversible) parts such that

$$
\varepsilon_{i j}=\varepsilon_{i j}^{e}+\varepsilon_{i j}^{p}, \varepsilon_{k k}^{p}=0
$$

with no elastic or inelastic strain occurring in the stress-free virgin state. Every material element in its virgin state is assumed to be isotropic. Constancy of volume due to inelastic deformation requires the trace of inelastic strain to be zero valued. Here repeated Latin indicies are summed from 1 to 3 in the usual manner.

The constitutive equation characterizing the thermoelastic behavior of an isotropic continuum is given by

$$
\varepsilon_{i j}^{e}=\frac{1+v}{E} \sigma_{i j}-\left(\frac{v}{E} \sigma_{k k}-\alpha \Delta T\right) \delta_{i j}
$$

where $\sigma_{i j}$ is the Cauchy stress, and $T_{0}$ is the reference temperature with $\Delta T=T-T_{0}$. Here $E$ is the elastic modulus, $v$ is the Poisson ratio, and $\alpha$ is the coefficient of thermal expansion. The Kronecker delta $\delta_{i j}$ has either the value 1 whenever $i=j$ or the value 0 whenever $i \neq j$.

The stress dependence of inelastic flow is strongly influenced by the material's anisotropy, which may be either inherent, or flow-induced, or both. In this paper only flowinduced anisotropy is addressed, and it is introduced after the manner of Prager (1949). This is done through an internal variable $B_{i j}$ called the back (internal or equilibrium) stress, which is a symmetric and traceless (i.e., deviatoric) tensor. To assure material isotropy in the virgin state, $B_{i j}[$ virgin state $]=0$. Square brackets $[\cdot]$ are used herein to denote "function of".

von Mises (1928) introduced the concept of an inclastic potential $F$ for describing the flow of inelastic strain, which we write here as

$$
\dot{\varepsilon}_{i j}^{p}=\left|\dot{\varepsilon}^{p}\right| \frac{\partial F}{\partial \sigma_{i j}}
$$

where $\partial F / \partial \sigma_{i j}$ defines the direction (in unit length) of inelastic straining, with $\left|\vec{\varepsilon}^{p}\right|$ providing its magnitude. The existence of this potential followed naturally from von Mises (1913) prior definition of yield, which is equivalent to introducing a second-invariant (or quadratic) norm that we write as 


$$
|I|=\sqrt{2 / 3 I_{i j} I_{j i}},|J|=\sqrt{3 / 2 J_{i j} J_{j i}}
$$

where $I_{i j}$ is any strain-like tensor (eg., $\left.\dot{\varepsilon}_{i j}^{p}\right)$, and $J_{i j}$ is any stress-like tensor (eg., $S_{i j}-B_{i j}$, where $S_{i j}=\sigma_{i j}-\left(\sigma_{k k} / 3\right) \delta_{i j}$ is the deviatoric stress). More recently, Rice (1971) demonstrated the physical existence of such a potential function based upon the mechanics and thermodynamics of dislocation slip. Most plastic and viscoplastic models take $F=|S-B|$, and therefore Eqn. 3 becomes

$$
\dot{\varepsilon}_{i j}^{p}=\frac{3}{2}\left|\dot{\varepsilon}^{p}\right| \frac{S_{i j}-B_{i j}}{|S-B|}
$$

which is compatible with the kinematic constructs proposed by Prager (1949) in his plasticity model. The choice of this particular inelastic potential, $F$, provides a reasonable approximation to the actual shapes of observed yield and flow surfaces ( $c f$. Clinard and Lacombe (1988)). The norms defined in Eqn. 4 are scaled for tension. They could have just as easily been scaled for shear by replacing the coefficients that appear under the radical signs with a 2 for $I_{i j}$ and a $1 / 2$ for $J_{i j}$.

Zener and Hollomon's (1944) experimental results demonstrate that the functional dependence for the magnitude of inelastic strain rate $\left|\dot{\varepsilon}^{p}\right|$ can, to a good approximation, be decomposed into the product of functions

$$
\left|\dot{\varepsilon}^{p}\left[T, S_{i j}, q_{\beta}\right]\right|=\theta[T] Z\left[S_{i j}, q_{\beta}\right] \geq 0
$$

where $\theta>0$ is the thermal diffusivity, $Z \geq 0$ is the Zener parameter, and $q_{\beta}$ is a set of independent internal state variables (to be defined shortly). The thermal diffusivity is often represented as an Arrhenius function of temperature, which is valid over a significant and specifible temperature range (cf. Miller (1976)). The functional dependence of the Zener parameter is of particular interest to us in this paper. This parameter is a temperature normalized function for the magnitude of inelastic strain rate.

In addition to the kinematic variable, or back stress $B_{i j}$, there are two isotropic variables introduced into the general structure of our Zener parameter; they are the drag (or friction) strength $D>0$ and the yield (or threshold) strength $Y \geq 0$. These three internal variables interact with the deviatoric stress $S_{i j}$ in such a way that the Zener parameter of Eqn. 6 is considered to have the following functional dependence

$$
\begin{gathered}
Z=Z\left[\frac{\leq|S-B|-Y\rangle}{D}\right]=Z\left[\frac{\left\langle\sigma^{\nu}\right\rangle}{D}\right]= \\
=\frac{\left|\dot{\varepsilon}^{p}\right|}{\theta} \geq 0
\end{gathered}
$$

with $Z[0]=0 \quad$ (cf. Freed and Chaboche (1989)). Here $\sigma^{\nu} \equiv|S-B|-Y$ is the viscous stress (or overstress) that govems inelastic material response. The Macauley bracket $\left\langle\sigma^{\nu}\right\rangle$ has either a value of 0 whenever $|S-B|<Y$ (defining the elastic or reversible domain) or a value of $\sigma^{v}$ whenever $|S-B|>Y$ (defining the inelastic or irreversible domain), with $|S-B|=Y$ establishing the yield surface. Viscoplasticity (a rate dependent theory) admits states within the yield surface, on the yield surface, and outside the yield surface. Plasticity (a rate independent theory), on the other hand, only admits states within and on the yield surface; it does not admit states outside the yield surface. The expression for the Zener parameter given above is very general, and includes three important special cases: $i)$ viscoplastic theories without a yicld surface (i.e., $Y=0$ ), ii) viscoplastic theories with a yield surface and no evolving drag strength (i.e., $D=$ constant , and iii) viscoplastic theories where the drag and yield strengths are equivalent (i.e., $D \equiv Y$ ).

\section{Steady-state creep}

Steady-state creep is an important limiting case of the viscoplastic representation given above. Let the creep rate $\dot{\varepsilon}_{i j}^{c}$ be an equivalent expression for the steady-state inelastic strain-rate $\dot{\varepsilon}_{i j s s}^{p}$, where the indicies ss denote steady state. Considering the inelastic potential $F_{s s}=|S|$, one obtains from Eqn. 3 the flow equation

$$
\dot{\varepsilon}_{i j}^{c}=\frac{3}{2}\left|\dot{\varepsilon}^{c}\right| \frac{S_{i j}}{|S|}
$$

which is a representation of Odqvist's (1936) theory for creep. For Eqn. 8 to in fact be a limiting case of Eqn. 5, it is necessary that the back siress $B_{i j}$ be coaxial with the deviatoric stress $S_{i j}$ at steady state, as observed in the experimental results of Blass and Findley (1971). This provides a physical constraint that every admissible evolution equation for back stress must satisfy.

Since steady-state creep is but a limiting case of viscoplasticity, the Zener-Hollomon (1944) decomposition of the magnitude of inelastic strain-rate, Eqn. 6, must also apply at steady state, but now there is no dependence on internal state, viz.

$$
\left|\dot{\varepsilon}^{c}\left[T, S_{i j}\right]\right|=\theta[T] Z_{s s}\left[S_{i j}\right]
$$

More specifically, we shall consider the stress dependence for the steady-state Zencr parameter as having the general form

$$
Z_{s s}=Z_{s s}\left[\frac{|S|}{A}\right]=\frac{\left|\dot{\varepsilon}^{c}\right|}{\theta} \geq 0
$$

with $Z_{s}[0]=0$, and where $A>0$ is a material constant. Common expressions for $Z_{s s}$ include: the power-law representation of Norton $(1929), Z_{s s}=(\mid S V A)^{n}$; the exponential representation of Dom (1954), $Z_{s s}=\exp (|S| / A)-1$; and the hyperbolic sine representation of Garofalo (1963), $Z_{s s}=\sinh ^{n}(|S| / A)$. Here the exponent $n$ has a value of about 5 (cf. Sherby $e t$ al. (1977)), and the value of $A$ will differ between these representations. The Norton relation applies to situations at lower stress states (typically associated with the higher temperature environments); whereas, the Dom relation applies to situations at higher stress states (typically associated with the more moderate temperature environments). The Garofalo relation relains both the Norton and Dorn relationships as its infimum and extremum, respectively.

\section{The hypothesis}

A hypothesis is put forth which enables one to formulate a theory of viscoplasticity that reduces, in closed form, to the classical theory of creep under conditions of steady state. This hypothesis is: the transient Zener parameter $Z$ has the same functional form as the steady-state Zener parameter $Z_{x}$, but with a different argument, viz.

$$
Z \equiv Z_{s s}\left[f\left[\frac{\langle|S-B|-Y>}{D}\right]\right] \geq 0
$$


with $f[0]=0$. This paper addresses the question: what are some of the admissible functional forms for $f$; however, it does not discuss in any detail what the functional forns for $Z_{s}$ might be. In addition to our hypothesis, it is also assumed that the back and deviatoric stresses are proportional at steady state; in particular, that

$$
|B|_{s s}=b|S|
$$

where $b \in(0,1)$ is the proportionality factor. Typically, $b=0.4$ and does not vary much from material to material ( $c f$. Gibeling and Nix (1982), and Freed and Walker (1989)). Equation 12 is a constraint relation that must be taken into account when developing an admissible evolution equation for back stress, as demonstrated in §IV.

At steady state, the arguments of Eqns. 10 and 11 must be equal to one another; therefore, using Eqn. 12, one obtains the identity

$$
f_{s s}=f\left[\frac{(1-b)|S|-Y_{s s}}{D_{s s}}\right]=\frac{|S|}{A}
$$

where we have also used the previous result (or constraint) that the back stress has to be coaxial with the applied stress at steady state.

Within the context of the two assumptions given in Eqns. 11 and 12, there are two theoretical restrictions that the transient function $f$ must satisfy for it to be physically sound, and therefore admissible, independent of whether or not it can correlate data. First, values for the isotropic variables in the virgin state, $D_{0}$ and $Y_{0}$. defined by the limits

$$
D_{0} \equiv \lim _{|s| \rightarrow 0} D_{s s} \text { and } Y_{0} \equiv \lim _{|S| \rightarrow 0} Y_{s s}
$$

must satisfy the inequalities $D_{0}>0$ and $Y_{0} \geq 0$. Physically, the virgin state is attained by annealing the material at a very high homologous temperature in the absence of extemal tractions. Second, steady-state values for these isotropic variables must monotonically increase with increasing stress at rates that are finite. Physically, Taylor (1934) determined that strength increases as the square root of dislocation density. Since the isotropic variables are phenomenological measures of dislocation density, their steady-state values should therefore monotonically increase with stress.

\section{TRANSIENT FUNCTIONS}

Ten different functional forms for the transient function $f$ of Eqn. 11 are presented in this section. Three viscoplastic models take the drag strength to be the isotropic variable. Four viscoplastic models take the yield strength to be the isotropic variable, after the manner of Chaboche (1977). And three viscoplastic models take the drag and yield strengths to be equivalent to one another, after the manner of Perzyna (1964). There are four different functional forms for $f$ applied to each of the three drag and yield strength representations; they are: linear, power law, exponential, and hyperbolic sine relationships. It is worth noting that the conclusions arrived at herein are independent of one's choice for the functional form for $Z_{s s}$, of which $f$ is its argument in our transformation to obtain $Z$ by hypothesis.

\section{Linear models}

The most common functional form for the transient function $f$ used in viscoplastic models today is a linear relationship. However, in none of these models, to the best of our knowledge, is there an attempt to constrain the evolution equarions so that their transient viscoplastic theory reduces, in closed form, to the classical theory of creep under steady-state conditions.

\section{I.I A drag model}

This model assumes that there is no yield surface (i.e., $Y=0$ ), and that the transient function $f$ is linear in its argument, viz.

$$
f=f\left[\frac{|S-B|}{D}\right]=\frac{|S-B|}{D}
$$

When the stcady-state form of this equation is combined with Eqn. 13, one obtains the constraint equation

$$
D_{s s}=A(1-b)
$$

implying that the steady-state value of drag strength is independent of the applied stress. This expression lacks physical interpretation since it does not monotonically increase with stress, and therefore Eqn. 15 is not acceptable on physical grounds.

\subsection{A Chaboche type yield model}

A Chaboche type yield surface is assumed in this linear model for the transient function $f$ (therefore, $D=$ constant $>0$ ) such that

$$
f=f\left[\frac{\leq|S-B|-Y>}{D}\right]=\frac{\langle|S-B|-Y\rangle}{D}
$$

which when combined with Eqn. 13 under steady-state conditions results in

$$
Y_{s s}=[A(1-b)-D] \frac{|S|}{A}
$$

Like the constraint equation for steady-state back stress given in Eqn. 12, this constraint equation for steady-state yield strength is also linear in stress. Linearity is advantageous when one goes about developing an admissible evolution equation for yield strength, as discussed in \$IV. In Eqn. 18, the drag strength $D$ is observed to be a parameter establishing proportionality between $Y_{s s}$ and $|S|$. Assuming that $Y_{s s}$ is about $5 \%$ of $|S|$, it follows then that $D \approx 0.9 A(1-b)$. Notice that whenever $D=A(1-b)$, one finds that $Y_{s s}=0$ independent of the stress state. But this is physically unacceptable for the same reason that the expression for $D_{z}$ is unacceptable in the previous model, i.e. it is in violation with the physics of Taylor (1934).

For the two theoretical restrictions, the value of yield strength in the virgin state, defined in Eqn. 14, is

$$
Y_{0}=0
$$

which is an acceptable result. Also, the derivative of Eqn. 18 with respect to stress given by

$$
\frac{d Y_{s s}}{d|S|}=1-b-\frac{D}{A} \geq 0
$$

is

non-negative and finite valued, provided that 
$D<A(1-b)$, which is an upper bound on $D$. Recall from the previous paragraph that $D \neq A(1-b)$ for physical reasons. Consequently, because both Eqns. 19 and 20 are satisfied, the yield surface model for $f$ given in Eqn. 17 is theoretically acceptable.

\subsection{A Perzyna type yield model}

A Perzyna type yield surface is assumed in this linear model for the transient function $f$ (therefore, $D \equiv Y$ ) such that

$$
f=f\left[\frac{\langle|S-B|-D\rangle}{D}\right]=\frac{\langle|S-B|-D\rangle}{D}
$$

At steady state, combining this relation with Eqn. 13 results in

$$
D_{s}=\frac{A(1-b)|S|}{A+|S|}
$$

which is a constraint equation that must be taken into account if one is to develop an admissible evolution equation for drag strength.

For the first theoretical restriction, the value of drag strength in the virgin state, defined in Eqn. 14, is determined to be

$$
D_{0}=0
$$

which is not acceptable. The second restriction requires that

$$
\frac{d D_{s s}}{d|S|}=\frac{A(1-b)}{A+|S|}\left[1-\frac{|S|}{A+|S|}\right] \geq 0
$$

which is acceptable, since it is non-negative and finite valued. Consequently, because Eqn. 23 is not acceptable, the yield surface model for $f$ given in Eqn. 21 is inadmissible.

\section{Power-Iaw models}

Suggested by the fact that steady-state and constantstructure (i.e. transient) creep data exhibit different power-law stress dependencies ( $c f$. Sherby et al. (1977)), Miller (1976) proposed that the transient function $f$ should be a power-law function. Here again, we shall consider both drag and yield strength models. We shall also propose a theoretically admissible, convoluted, yield strength model.

\section{I A drag model}

A power-law was used by Miller (1976) for $f$ in his original viscoplastic theory, wherein he also used Garofalo's (1963) hyperbolic sine relationship for $Z_{s s}$. Here the transient function $f$ (a power-law modeI with no yicld surface, i.e., $Y=0)$ is defined as

$$
f=f\left[\frac{|S-B|}{D}\right]=\left[\frac{|S-B|}{D}\right]^{m}
$$

where the exponent $m$ (the constant structure exponent divided by the steady-state exponent, see Fig. 1) has a typical value of $m \approx 1.5$. Like $b$, this typical value for $m$ does not vary much from material to material ( $c f$. Sherby et al. (1977)). It therefore follows from Eqns. 13 and 25 at steady-state that

$$
D_{s s}=A(1-b)\left(\frac{|S|}{A}\right)^{\frac{m-1}{m}}
$$

which is a constraint, like Eqn. 12, that must be taken into $\triangle$ SERVI-GRANT (1951)

GIBELING-NIX (1982)

- CONSTANT STRUCTURE

- STEADY STATE

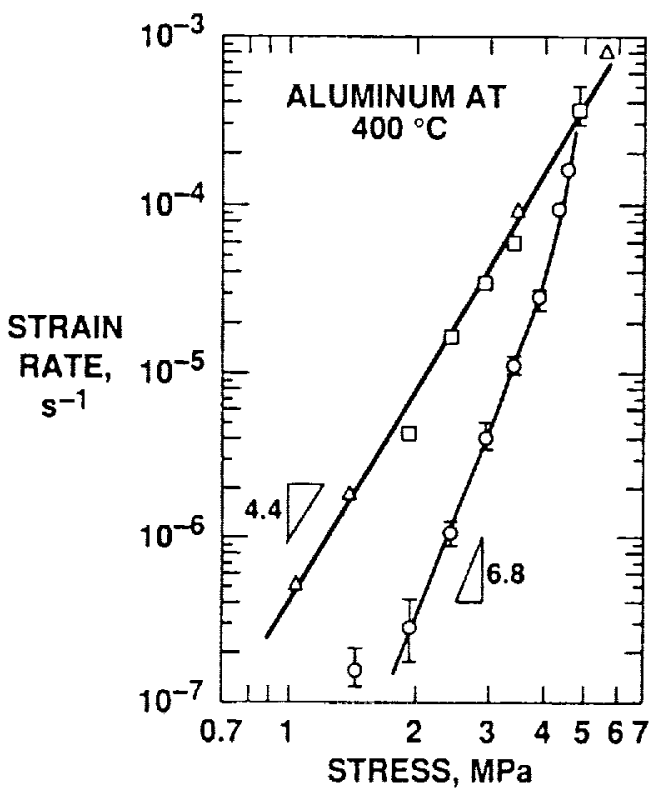

FIG. 1. Log stress vs. log strain-rate (power-law stress dependence) of steady-state and constant-structure creep data.

account if one is to develop an admissible evolution equation for drag strength ( $c f$. Miller (1976), and Freed and Walker (1989)). In the development of Miller's model, Eqn. 26 was first proposed (and substantiated by data from warm worked materials), from which Eqn. 12 was then derived.

For the first theoretical restriction, the value of drag strength in the virgin state, defined in Eqn. 14, has the value

$$
D_{0}=0
$$

which must be positive-valued because of physical arguments, but it is not. For the second theoretical restriction, the derivative of Eqn. 26 with respect to stress given by

$$
\frac{d D_{s s}}{d|S|}=(1-b) \frac{(m-1)}{m}\left(\frac{A}{|S|}\right)^{\frac{1}{m}} \geq 0
$$

must be non-negative valued, which it is, but it is not finite valued. Even though this derivative implies that the steadystate value of drag strength monotonically increases with increasing stress, the slope becomes infinite as the stress approaches zero, which is not acceptable. Since neither Eqns. 27 or 28 are desirable results, the drag strength model for $f$ given in Eqn. 25, although simple, is not considered to be acceptable.

The remaining models for the transient function $f$ have not appeared in the literature, to the best of our knowledge.

\subsection{A Chaboche type yield model}

Again we take the transient function $f$ to be a powerlaw of its argument, but this time we consider $D=$ constant $>0$, thereby resulting in a Chaboche type yield 
surface model

$$
f=f\left[\frac{\langle|S-B|-Y>}{D}\right]=\left[\frac{\leq|S-B|-Y>}{D}\right]^{m}
$$

where once again the exponent has a typical value of $m \approx 1.5$. By combining Eqns. 13 and 29 under steady-state conditions, one obtains the relationship

$$
Y_{s s}=A(1-b) \frac{|S|}{A}-D\left[\frac{|S|}{A}\right]^{\frac{1}{m}}
$$

which, like Eqn. 26 of the previous model, is a constraint that must be taken into account if one is to develop an admissible evolution equation for yield strength.

For the first theoretical restriction, the value of yield strength in the virgin state, defined in Eqn. 14, has the value

$$
Y_{0}=0
$$

which must be non-negative valued, and it is. However, the derivative of Eqn. 30 with respect to stress (the second theoretical restriction) given by

$$
\frac{d Y_{v}}{d|S|}=1-b-\frac{D}{m A}\left(\frac{A}{|S|}\right)^{\frac{m-1}{m}}
$$

is negative valued over the stress range from $|S|=0$ to $|S|=A(D /(m A(1-b)))^{m /(m-1)}$. This implies that the yield strength has negative values (because of Eqn. 31 ) in a specifable neighborhood of the virgin state, which obviously is not correct. Therefore, the yield strength model for $f$ given in Eqn. 29 is not acceptable, either.

\subsection{A Perzyna type yield madel}

Here a Perzyna type yield surface model is considered where the transient function $f$ is a power-law model, i.e.

$$
f=f\left[\frac{\leq|S-B|-D\rangle}{D}\right]=\left[\frac{\leq|S-B|-D\rangle}{D}\right)^{m}
$$

where the exponent $m$ has the same value as before. Therefore, by combining the steady-state form of Eqn. 33 with Eqn. 13 , one obtains

$$
D_{s}=\frac{(1-b)|S|}{1+\left(\frac{|S|}{A}\right)^{\frac{1}{m}}}
$$

as the constraint equation for steady-state drag strength.

For the two theoretical restrictions, drag strength in the virgin state, as defined by Eqn. 14, has the value

$$
D_{0}=0
$$

which is unacceptable, because it is zero valued. However, the derivative of steady-state drag strength with respect to stress, given by

$$
\frac{d D_{s s}}{d|S|}=\frac{1-b}{1+\left(\frac{|S|}{A}\right)^{\frac{1}{m}}} \times
$$

$$
\times\left\{1-\frac{\left[\frac{|S|}{A}\right]^{\frac{1}{m}}}{m\left\{1+\left[\frac{|S|}{A}\right]^{\frac{1}{m}}\right\}}\right\} \geq 0
$$

is acceptable, because it is non-negative and finite valued. Nevertheless, since the first restriction is not satisfied, the yield strength model for $f$ given in Eqn. 33 is inadmissible, too.

\subsection{A convoluted yield madel}

In this model, we convolute the yield strength formulation in Eqn. 29 in such a way that there is no stress range over which $d Y_{s s} / d|S|$ is negative valued; thereby, resulting in a theoretically admissible model. Considering the transient function

$$
\begin{gathered}
f=f[|S-B|, D, Y]= \\
=\left[\frac{A(1-b)}{|S-B|}\right]^{m-1}\left[\frac{\leq|S-B|-Y>}{D}\right]^{m}
\end{gathered}
$$

one then obtains the following expression for the steady-state yield strength, i.e.

$$
Y_{s s}=[A(1-b)-D) \frac{|S|}{A}
$$

where Eqn. 12, and the fact that the back and applied stresses must be coaxial at steady state, were used in its derivation. This is the same expression for the steady-state value of yield strength given earlier in Eqn. 18; hence, the two theoretical restrictions given in Eqns. 19 and 20 also apply here. Consequently, the yield surface model for $f$ defined in Eqn. 37 is theoretically acceptable, and it is the only power-law expression for $f$ of those considered herein that is acceptable. Because $D$ is near in value to $A(1-b)$, Eqn. 37 will not be very sensitive to the value of $m$, and therefore a simplifying value of $m=2$ is suggested.

\section{Exponential models}

Gibeling and Nix (1982) performed a detailed experimental study on aluminum to answer the following question: what is the appropriate stress dependence of transient creep? Their results are presented in Fig. 1. Because these data are for one temperature, the recorded strain-rate values are proportional to their associated Zener values. Over the stress range considered therein, the steady-state creep rates are adequately described by a power-law (or Norton (1929)) stress dependence with an exponent of 4.4, as shown in Fig. 1. The constant structure (or transient) data can also be described by a power-law stress dependence with an exponent of 6.8, but only over a limited range in stress, as also seen in Fig. 1. 1 This results in a value for the exponent $m$ in Eqns. 25,29 and 33

1 The constant structure data reported in Figs. 1 and 2 are from isothemal experiments that began as creep tests at a fixed level of stress $\left(4.82 \mathrm{MPa}\right.$ at $\left.400^{\circ} \mathrm{C}\right)$, and which were crept into steady state (21 percent true strain). At that point, these specimens underwent a quick step reduction in stress (each of a different degree), and the ensuing strain rates were recorded. 
of $m=6.8 / 4.4 \approx 1.5$. However, as is also shown in this figure, the constant structure exponent increases in value to 9 or 10 for small stress reductions. Consequently, the transient data of Gibeling and Nix (1982) are better described by an exponential relationship, as shown in Fig. 2. Later, Nix and Gibeling (1985) gave a physical interpretation for the exponential stress dependence of constant structure.

\section{$3.1 \mathrm{~A}$ drag model}

Taking the transient function $f$ to be an exponential of its argument, in accordance with Figs. 1 and 2, and assuming that there is no yield surface (i.e., $Y=0$ ), results in the model

$$
f=f\left[\frac{|S-B|}{D}\right]=\exp \left[\frac{|S-B|}{D}\right]-1
$$

By combining Eqns. 13 and 39 under steady-state conditions, one obtains the result

$$
D_{s s}=\frac{A(1-b) \frac{|S|}{A}}{\ln \left(1+\frac{|S|}{A}\right)}
$$

which is a constraint that must be taken into account if one is to develop an admissible evolution equation for drag strength. Such a development requires taking the inverse of this function, i.e. expressing $|S| A \mid$ as a function of $D_{s s}$, as demonstrated in §IV.

The value of the drag strength in the virgin state, as defined in Eqn. 14, is determined to be

$$
D_{0}=A(1-b)
$$

which is positive valued, as it must be. Likewise, the derivative of Eqn. 40 with respect to stress given by

$$
\begin{aligned}
& \frac{d D_{s s}}{d|S|}=\frac{1-b}{\ln \left[1+\frac{|S|}{A}\right]} \times \\
& \times\left\{1-\frac{\frac{|S|}{A}}{\left[1+\frac{|S|}{A}\right] \ln \left[1+\frac{|S|}{A}\right]}\right\} \geq 0
\end{aligned}
$$

is non-negative and finite valued, as it must be. Since both theoretical restrictions are satisfied, the drag strength model for $f$ given in Eqn. 39 is admissible.

\subsection{A Chaboche type yield model}

Here a transient function $f$ is considered which contains a Chaboche type yield surface where the drag strength does not evolve (i.e. $D=$ constant $>0$ ), and is an exponential of its argument, viz.

$$
\begin{aligned}
& f=f\left[\frac{\leq|S-B|-Y\rangle}{D}\right]= \\
& =\exp \left[\frac{\langle|S-B|-Y\rangle}{D}\right]-1
\end{aligned}
$$
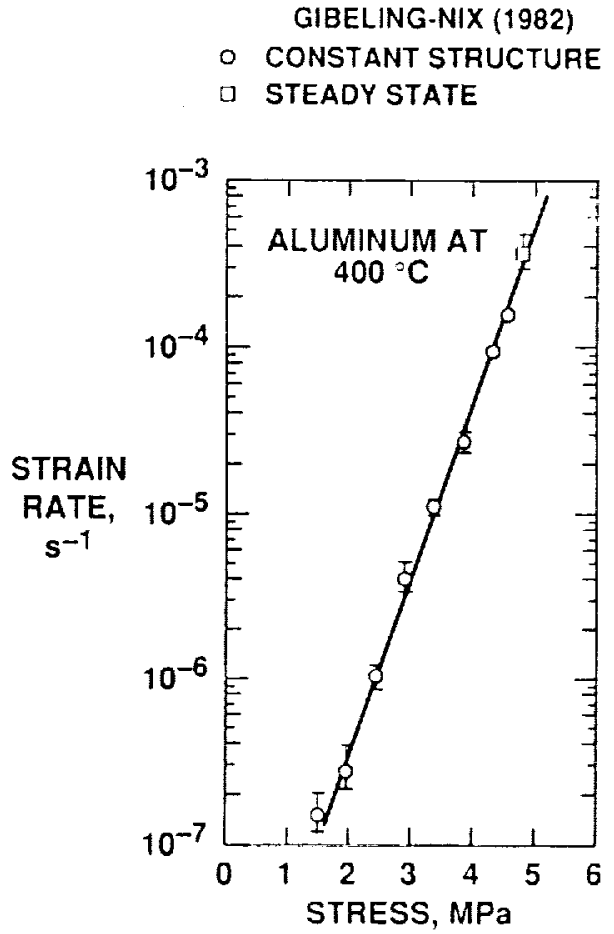

FIG. 2. Stress vs. log strain-rate (exponential stress dependence) of steady-state and constant-structure creep data.

By combining Eqns. 13 and 43 under steady-state conditions, one obtains the relationship

$$
Y_{s s}=A(1-b) \frac{|S|}{A}-D \ln \left(1+\frac{|S|}{A}\right)
$$

which is a constraint that must be taken into account if one is to develop an admissible evolution equation for yield strength. Such a development requires taking the inverse of this function, which is tricky, but can be done, as demonstrated in \$IV.

For the theoretical restrictions, the value of yield strength in the virgin state, as defined in Eqn. 14, is

$$
Y_{0}=0
$$

which is non-negative valued, as it must be. Also, the derivative of Eqn. 44 with respect to stress given by

$$
\frac{d Y_{s s}}{d|S|}=1-b-\frac{D}{A+|S|} \geq 0
$$

is positive and finite valued, provided that $D \leq A(1-b)$, which is an upper bound on $D$. Consequently, the yield strength model for $f$ presented in Eqn. 43 is theoretically acceptable, too.

\subsection{A Perzyna type yield model}

Finally, we consider a transient function $f$ that contains a Perzyna type yield surface (i.e. $D \equiv Y$ ), and which is an exponential of its argurnent, viz. 


$$
\begin{aligned}
& f=f\left[\frac{\leq|S-B|-D>}{D}\right]= \\
& =\exp \left[\frac{\leq|S-B|-D>}{D}\right]-1
\end{aligned}
$$

By combining Eqns. 13 and 47 under steady-state conditions, one obtains

$$
D_{s}=\frac{(1-b)|S|}{1+\ln \left(1+\frac{|S|}{A}\right)}
$$

as the constraint equation for steady-state drag strength.

The first theoretical restriction requires the drag strength to be positive valued in the virgin state, as defined in Eqn. 14, but

$$
D_{0}=0
$$

which is not acceptable, because it is zero valued. The second theoretical restriction requires the derivative of steady-state drag strength with respect to stress to be non-negative and finite valued, i.e.

$$
\begin{aligned}
& \frac{d D_{s s}}{d|S|}=\frac{1-b}{1+\ln \left(1+\frac{|S|}{A}\right)} \times \\
& \times\left\{1-\frac{\frac{|S|}{A}}{\left[1+\frac{|S|}{A}\right]\left\{1+\ln \left[1+\frac{|S|}{A}\right]\right\}}\right\} \geq 0
\end{aligned}
$$

which is acceptable. However, because the first restriction is not satisfied, the yield strength model for $f$ given in Eqn. 47 is inadmissible.

\section{Hyperbolic sine models}

Upon examining the constant structure data in Fig. 1, one notices that the shape of this curve is much like that of steady-state creep curves where the stresses exceed power-law breakdown, and which Garofalo (1963) modeled using a hyperbolic sine raised to a power. We investigated (but do not report the details of, because of space limitations) both drag and yield-surface models where hyperbolic sines of these arguments were applied, and which were then raised to a power (akin to Garofalo's creep equation). None of these models satisfy both of the theoretical restrictions necessary for them to be admissible transient functions. This is not suprising, since they reduce to power-law models as the stress tends toward zero, and consequently, they share the same deficiences that our first three power-law models possess.

\section{A selection}

Recent experimental results from Krempl (1987) on type 304 stainless steel at room temperature - presented here in Fig. 3 - indicate that isotropic hardening is better represented by a Chaboche type yield strength parameter than by either a drag strength or a Perzyna type yield strength parameter. Notice that the changes in stress across the jumps in engineering strain-

\section{TYPE 304 STAINLESS STEEL AT $21^{\circ} \mathrm{C}$ KREMPL (1987)}

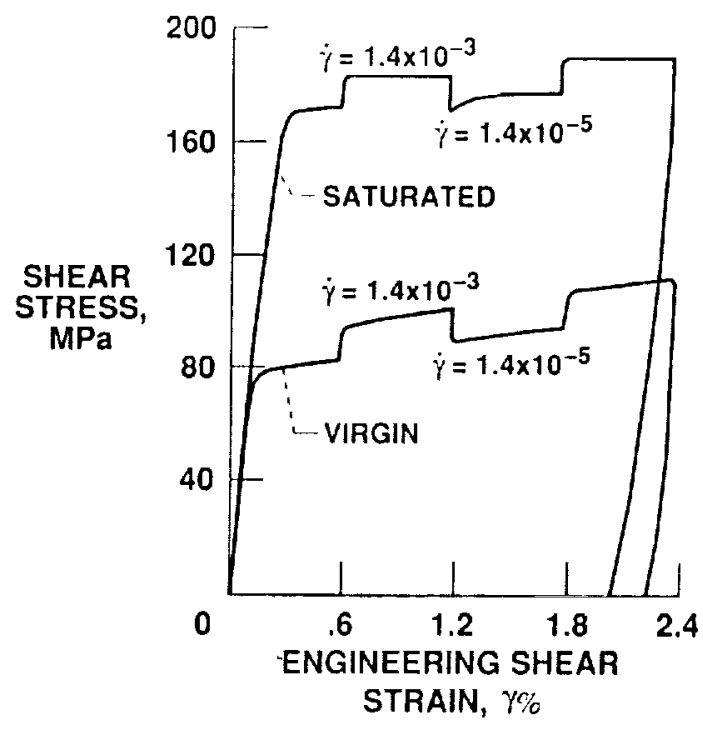
dening.

FIG. 3. Material strain-rate sensitivity is independent of har-

rate are, to a good approximation, the same in both the virgin and saturated states. In other words, the observed rate dependence is independent of hardening; a result that a Chaboche type yield surface model would predict, but one that a drag strength model or a Perzyna type yield strength model cannot predict.

Quite frankly, the authors were initially suprised that yield surface models (in particular, Eqns. 17, 37 and 43) would be preferred over ones without a yield surface (since only Eqn. 39 is theoretically admissible). Curiously, only Chaboche type yield surface models are theoretically admissible; Perzyna type yield surface models are not, at least within the constraints of our hypothesis. Furthermore, these viscoplastic yield surface models have been shown to reduce at steady state to creep models where yield surfaces are not present.

Of the three yield strength models for the transient function $f$ that are theoretically admissible, i.e. Eqns. 17, 37 and 43, the authors prefer the linear model over the convoluted power-law and exponential models for reasons of simplicity and ease in interpretation. The linear model has the simplest overall form. Also, the linear and convoluted power-law models are simpler than the exponential model when it comes to constructing admissible evolution equations for the yield strength, which is discussed at greater length in the next section. The linear and convoluted power-law models also have a simpler interpretation of what the drag strength is, and what its approximate value should be. All three models predict roughly the same response for the constant structure data of Gibeling and Nix (1982), as illustrated in Fig. 4, because the Zener parameter $Z=Z_{s s}[f]$ is a strong function of its argument $f$, whereas $f$ is a relatively weak function of its argument in these models. 2 This indicates that the internal state variables, 


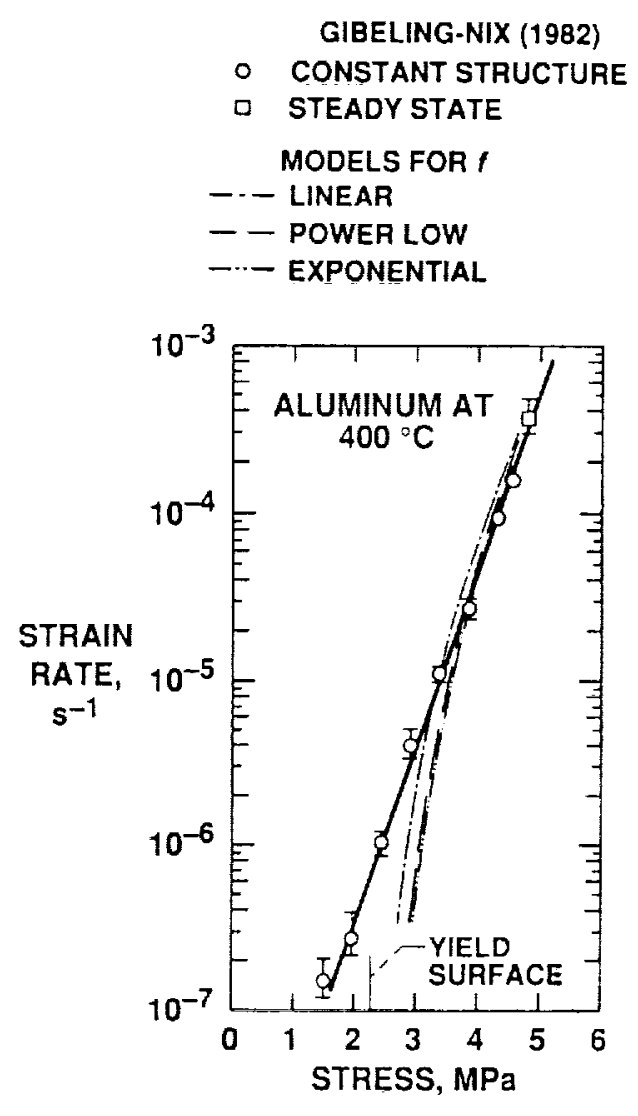

TIG. 4. Constant-structure creep predictions are, to a good approximation, independent of one's choice for the transient function $f$.

$B_{i j}$ and $Y$, have more to do with an accurate representation of constant structure data than does the functional form for the transient function $f$; therefore, the simplest function for $f$ is the best function for $f$, i.e. Eqn. 17 ,

$$
f=f\left[\frac{\langle|S-B|-Y\rangle}{D}\right]=\frac{\langle|S-B|-Y\rangle}{D}
$$

is the transient function that we select. One also observes in Fig. 4 that the predicted inelastic strain-rate of all three models deviates from the data by tending towards zero as the stress approaches the yield surface (a value of about $2.2 \mathrm{MPa}$ in these models). Gibeling and Nix experimentally determined that the back stress has a value between 1.4 and $2 \mathrm{MPa}$ (and therefore, $b=0.4$ ); in fact, their datum point at $1.4 \mathrm{MPa}$ displayed in Figs. 1, 2 and 4 is associated with a negative or reversed strain-rate.

\section{THERMAL RECOVERY}

The internal state variables of viscoplasticity (back stress, drag strength, and yield strength) evolve phenomenolog-

\footnotetext{
2 Here values used for the material constants are: $A=29.9 \mathrm{MPa}, \quad b=0.4, D=0.9 A(1-b)=16.1 \mathrm{MPa}, m=2$ and $n=4.4$, where Norton's (1929) relationship $\dot{\varepsilon}^{c}=(\sigma / A)^{n}$ is used for the steady-state $Z$ ener parameter $Z_{s s}$ with $\theta=1$.
}

ically via a hardening vs. recovery format ( $c f$. Freed and Chaboche (1989)). By definition, steady state exists when the effect due to hardening mechanisms is exactly cancelled out by the effect due to recovery mechanisms for all the internal variables. In the discussion that follows, thermal recovery will be the only recovery mechanism considered. In a future paper, the authors will address the more subtle situation where both static (thermally induced) and dynamic (strain induced) recovery mechanisms are present. Our purpose here is to only illustrate how one goes about specifying the recovery functions in such a manner that the transient Zener parameter of Eqn. 7 reduces to that of Eqn. 10 under conditions of steady state.

A hardening vs. thermal-recovery evolution format for the back stress is given by

$$
\dot{B}_{i j}=\frac{2}{3} H\left[\dot{\varepsilon}_{i j}^{p}-\frac{3}{2} \theta[T] R\left[B_{w j}\right] \frac{B_{i j}}{|B|}\right]
$$

where $H>0$ is the hardening modulus, and $R \geq 0$ is the thermal recovery function. By definition, $\dot{B}_{i j}=0$ at steady state, and consequently, the back stress is coaxial with the applied stress at steady state, as required. It then follows from Eqns. 5 to 11 and Eqn. 51 , that $R\left[B_{i j, s}\right]=Z_{w}\left[S_{i j}\right]$ at steady state. Hence, with a relationship existing between back stress and deviatoric stress at steady state in the form of Eqn. 12, one readily obtains the following expression for the thermal recovery of back stress, i.e.

$$
R=Z_{s s}\left[\frac{|B|}{b A}\right]
$$

where the subscript $s s$ can be dropped from $|B|_{s s}$ (which would otherwise appear in the argument of $Z_{s s}$ ), because steady state is only a special case, and therefore, the same equation must be capable of handling transient situations as well. This functional form for the thermal recovery of back stress is required if our theory of viscoplasticity is to reduce to the classical theory of creep under steady-state conditions.

A similar hardening vs. thermal-recovery evolution format for the drag and yield strengths is given by

$$
\dot{K}=h\left[\left|\dot{\varepsilon}^{p}\right|-\theta[T] r[K]\right)
$$

where $K \in\{D, Y\}, h>0$ is the hardening modulus, and $r \geq 0$ is the thermal recovery function. By definition, $\dot{K}=0$ at steady state, and then from Eqns. 6 to 7,9 to 11 and 53, it follows that $r\left[K_{s s}\right]=Z_{s s}\left[S_{i j}\right]$. Or, as a more general expression, one obtains

$$
r[K]=Z_{s s}[\pi[K]]
$$

where the functional form of $\pi_{s v}[K] \equiv|S| / A$ depends upon the functional form of the transient function $f$.

The function $\pi[Y]$ is the same for both the linear and convoluted power-law models that use the Chaboche type yield surface, i.e. Eqns. 17 and 37, because both of these models have the same relationship between stress and yield strength at sleady state, viz. Eqns. 18 and 38, and therefore

$$
\pi=\frac{Y}{A(1-b)-D}
$$

for these two models. This functional form for the thermal recovery of yield strength is required if our selected theory of 
viscoplasticity (i.e. the linear model for $f$ ) is to reduce to the classical theory of creep under steady-state conditions.

If one were to develop a viscoplastic theory with a Chaboche type yield surface using the exponential model for $f$, Eqn. 43 , then the function $\pi[Y]$ is not so easily obtained. To determine its value, it is necessary to solve Eqn. 44 for ISVA, which does not appear to have a closed form solution. However, an approximate solution can be obtained by writing Eqn. 44 as

$$
y=a x-D \ln (1+x)
$$

where $y=Y_{s s}, \quad x=|S| / A \equiv \pi[Y]$ and $a=A(1-b)$. We can invert this expression by considering

$$
x=\frac{1}{a} y+\delta[x]
$$

where $\delta$ is a small perturbation given by

$$
\delta[x]=\frac{D}{a} \ln (1+x)=\frac{D}{a} \ln \left[1+\frac{1}{a} y+\delta[x]\right)(58)
$$

which can be determined to whatever degree of accuracy that one desires via repeated substitution. Three or so repeated substitutions should give enough accuracy for engineering purposes. The smaller the value of $y$, the larger the error is in $x \equiv \pi$.

Similarly, if one were to develop a viscoplastic theory without a yield surface using the exponential model for $f$ given in Eqn. 39 (the only theoretically acceptable drag strength model, herein), then the function $\pi[D]$ does not appear to have a closed form solution, either. However, an approximate inverse to Eqn. 40 can be obtained by first expanding it in a Taylor series, i.e. $y=a+1 / 2 a x+\cdots$, and then writing Eqn. 40 as

$$
\begin{gathered}
y=a+1 / 2 a x-\left[a+1 / 2 a x-\frac{a x}{\ln (1+x)}\right] \\
=a[1+1 / 2 x-\delta[x])
\end{gathered}
$$

where $y=D_{s s}, x=|S| / A \equiv \pi[D]$ and $a=A(1-b)$, and where

$$
\delta[x]=1+1 / 2 x-\frac{x}{\ln (1+x)}
$$

which is a perturbation to the function $y$. Therefore by inverting Eqn. 59, one obtains the desired expression

$$
x=2\left(\frac{1}{a} y-1+\delta[x]\right)
$$

which can be solved to whatever degree of accuracy that one desires via repeated substitution of Eqn. 61 into Eqn. 60, like the repeated substitution method of the previous paragraph. The technique presented in this paragraph for taking the inverse of a function can be used to obtain an approximate inverse of almost any function where a closed form inverse does not exist.

\section{CONCLUSIONS}

The objectives of this paper were to determine which isotropic variable, i.e. drag strength or yield strength, is the most appropriate for viscoplasticity, and to develop a viscoplastic theory that reduces, in closed form, to the classical theory of creep. Both of these objectives have been met.

Herein, we proposed a hypothesis that the transient Zener parameter $Z$ has the same functional form as the steady-state Zener parameter $Z_{s s}$, but with an argument $f$ instead of $|S| / A$ such that $f_{s}=|S| A$. Linear, power law, exponential, and hyperbolic sine relationships have all been considered as functional forms for the transient function $f$. For each of these relationships, we considered a drag strength model and two yield strength models (of both the Chaboche and Perzyna types). These functions for $f$ must satisfy two restrictions to be theoretically admissible. Three of the Chaboche type yield strength models and one of the drag strength models are shown to be theoretically admissible within the constraints of our hypothesis.

Experimental results of Krempl (1987) suggest that yield strength is the more physically correct description of isotropic hardening. The three admissible yield strength models are shown to all produce similar constant structure responses, because the Zener parameter $Z=Z_{s s}[f]$ is a strong function of its argument $f$, whereas $f$ is a relatively weak function of its argument. This implies that the intemal variables have more to do with constant structure (or transient) behavior than does the functional form of $f$. For this reason, the simplest function for $f$ (a linear yield strength model) is selected as being the best choice.

An important consequence of our hypothesis is that it enables one to determine functional forms for the thermal recovery functions in the evolution equations of the internal state variables (i.e., the back stress, drag strength and yield strength). These forms ensure that the viscoplastic theory reduces to the classical theory of creep under steady-state conditions.

\section{REFERENCES}

Blass, J J, and Findley, W N (1971), Shon-time, biaxial creep of an aluminum alloy with abrupt changes of ternperature and state of stress, $J$ Appl Mech 38, 489-501.

Chaboche, J-L (1977), Viscoplastic constitutive oquations for the description of cyclic and anisotropic behavior of metals, Bull Acad Polon Sci, Ser Sci Tech. nol $25,33-42$.

Clinard, J A, and Lacombe, C (1988), Detemination of multiaxial flow surfaces at elevated temperatures using the concept of dissipation potential, ORNL TM-10787.

Dom, J E (1954). Some fundamental experiments on high temperature creep, $J$ Mech Phys Solids 3, 85-116.

Freed, A D, and Chaboche, J-L (1989), Viscoplasticity: themodyramic formulation. NASA TM-102388; submitted to J Appl Mech.

Freed, A D, and Walker, K P (1989), Refinements in a viscoplastic model, in Visco-Plastic Behavior of New Materials, PVP vol 184 and MD vol 17, eds D Hui and T I Kozik, ASME, New York, 1-9.

Garofalo, F (1963), An empirical relation defining the stress dependence of minimum creep rate in metals, Trans AIME 227, 351-356.

Gibeling, J C, and Nix, W D (1982), Anomalous and constant substructure creep transients in pure aluminum, in Streng th of Metals and Alloys, ICSMA 6, vol 2 ad R C Gifkins, Pergamon Press, Oxford, 613-618.

Krempl, E (1987), Models of viscoplasticity: some comments on equilibrium (back) stress and drag stress, Acta Mech 69, 25-42.

Miller, A (1976), An inelastic constitutive model for monotonic, cyelic, and crep deformation: part I - equations development and amalytical procedures, $J$ Eng Mater Technol 98, 97-105.

von Mises, $R \vee(1913)$, Mechanik des festen körper in plastisch-deformablen zustand, Nachr Ges Wiss, Goettingen, Math Phys Kl, 582-592. 
von Mises, $R$ v (1928), Mechanik der plastischen formändenung von kristallen, Zeit Ang Math Mech 8, 161-185.

Nix, W D, and Gibeling, J C (1985), Mechanisms of time-dependent flow and fracture in metsls, in Flow and fracture at clevated temperalures, Metals/materials technology scries, ASM, Metals Park, OH, 1-63.

Nonon, F H (1929), Creep of steel at high temperatures, McGraw-Hill, New York.

Odqvist, F K G (1936). Theory of creep under the action of combined stresses with applications to high tempenture machinery, Proc $R$ Swed Inst Eng Res 141, 1-31.

Perzyna, P (1964), On the constitutive equitons for work-hardening and rate sensitive plastic materials, Bull Acad Polon Sci, Sér Sci Technol 12, 199-206.
Prager, W (1949), Recent developments in the mathematical theory of plasticity, J Appl Phys 20, 235-241.

Rice, J R (1971), Inelastic constitutive relastions for solids: an internal-variable theory and its application to metal plasticity, $J$ Mech Phys Solids 19, 433-455.

Servi, I S, and Grant, N I (1951), Creep and stress rupture behavior of aluminum as a function of purity, $J$. Metals 3, 909-916.

Sherby, O D, Klund, R H, and Miller, A K (1977), Flow stress, subgrain size, and subgrain stability at elevated temperature, Met Trans 8A, 843-850.

Taylor, G 1 (1934), The mechanism of plastic deformation of crystals: part I theoretical, Proc Roy Soc (London) A145, 362-387.

Zener, C, and Hollomon, J H (1944), Effect of strain rate upon plastic flow of steel, $J$ Appl Phys 15, 22-32. 


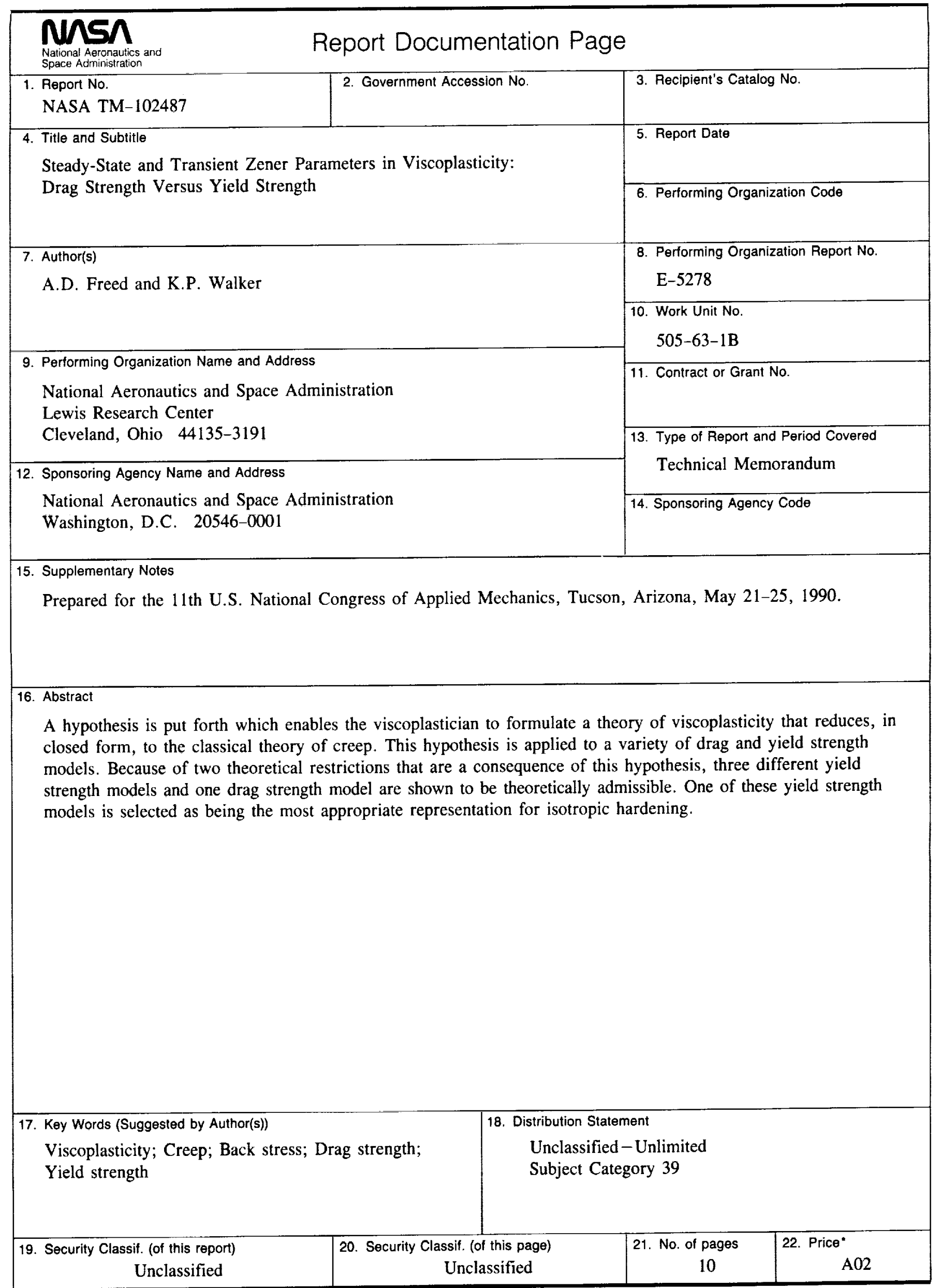

